\title{
Why the Converse Consequence Condition cannot be accepted
}

\section{LUCA MORETTI}

Three general confirmation principles discussed by Hempel (1965) are the following:

Converse Consequence Condition (CCC) If an observation statement $E$ confirms a hypothesis $H$ and if another hypothesis $H^{*}$ entails $H$, then $E$ confirms $H^{*}$.

Special Consequence Condition (SCC) If an observation statement $E$ confirms a hypothesis $H$, then $E$ confirms any of $H$ 's logical consequences.

Entailment Condition (EC) If an observation statement $E$ entails another statement $E^{*}$, then $E$ confirms $E^{*}$.

Since the conjunction of the above three principles entails the paradoxical consequence that any observation statement confirms any hypothesis (see Le Morvan 1999: 449), Hempel notoriously chose to preserve (SCC) and (EC), while rejecting (CCC). Yet, as Le Morvan (1999) has pointed out, one could think of preserving (CCC) by rejecting either (a) both (EC) and (SCC) or (b) only (EC) or (c) only (SCC). In fact, in none of these alternatives does the condition entailing the paradox appear satisfied. Trying to save (CCC) is not pointless since, as Glymour has emphasised, the Converse Consequence Condition 'has had an undying popularity, and attempts to make it work still continue' (Glymour 1980: $30)$.

Le Morvan (1999) has however argued that, when the choice among general confirmation principles is just about (CCC), (SCC) and (EC), since none of the three alternatives (a)-(c) is actually acceptable, it is (CCC) that must be rejected. In this paper, I aim to strengthen this argument and to make it definitive.

To begin with, according to Le Morvan, since both (EC) and (SCC) are quite intuitive, the alternative (a), which imposes the rejection of both of them, 'may strike many as a too high price to pay' (1999: 450), and thus is scarcely acceptable. I find this consideration straightforward. Le Morvan has convincingly shown that also the alternative (b) is unworkable, as the simple conjunction of (CCC) and (SCC) entails the paradoxical consequence that, if an observation statement confirms a hypothesis, then the observation statement confirms any hypothesis (see 1999: 450). ${ }^{1}$ Finally,

1 Le Morvan attributes the proof to an anonymous referee of Philosophy of Science. 
Le Morvan has argued that the alternative (c) is disastrous too, as one can deduce the paradoxical consequence that any observation statement confirms any hypothesis using only (EC) and (CCC).

Le Morvan's proof against the alternative (c) rests however upon certain assumptions proper to Hempel's conception of qualitative confirmation that many methodologists would reject. ${ }^{2}$ Such methodologists could therefore decide to preserve (CCC) in conjunction with (EC), while rejecting (SCC), without facing any immediate difficulty. Accordingly, since the choice to reject (CCC) is in part based on questionable assumptions, such a choice can be argued not to be really justified.

As I will show, it is however possible to reinforce Le Morvan's contention that the alternative (c) is unworkable by providing a general argument, which is independent of Hempel's specific conception of confirmation, to the effect that the simple conjunction of (EC) and (CCC) entails a disastrous consequence.

Le Morvan's proof is the following (cf. 1999: 449-50):

(1) Let $E^{*}$ be a tautology.

(2) Any observation statement $E$ entails $E^{*}$.

(Logical truth)

(Logical truth)

(3) Any hypothesis $H$ entails $E^{*}$.

(4) Any $E$ confirms $E^{*}$.

(5) Any $E$ confirms any $H$.

As Le Morvan himself recognizes, a weak point of his proof is the step (4). For many (or, probably, most) methodologists will claim that, since $E^{*}$ is by assumption a tautology, it cannot be confirmed by any statement at all. They will urge that (EC) should obviously be intended as stating that:

(EC*) If a statement $E$ entails a non-tautological statement $E^{*}$, then $E$ confirms $E^{*}$.

These methodologists will finally object that, on the grounds of (EC*), the above proof is incorrect.

Le Morvan's reply is that accepting (EC*) would come 'at the cost of repudiating some key aspects of the Hempelian conception of qualitative confirmation' (1999: 451), which entail that tautologies like $\forall x(P x \supset P x)$ and $\exists x(P x) \vee \sim \exists x(P x)$ are confirmed by any observation statement (cf. 1999: 452).

In this short paper, I cannot go deeper into the criticism of such aspects of Hempel's view of confirmation. I will only emphasise that they could be rejected on an intuitive basis, as they clash with the natural assumption

2 Notice that (CCC), (SCC) and (EC) are quite general and their intuitive plausibility does not depend on any specific assumption about qualitative confirmation. 
that, since tautological statements have no empirical content, they cannot be subject to empirical confirmation (or disconfirmation).

What Le Morvan has not noticed is that there is a simple way to reformulate his proof whose validity does not rest on the implausible claim that tautologies can be confirmed by evidence. Here is the new formulation:

(6) Let $E$ be any non-tautological observation statement and let $H$ be any hypothesis such that $E \vee H$ is not a tautology.

(7) $E$ entails $E \vee H$.

(8) $H$ entails $E \vee H$.

(9) $E$ confirms $E \vee H$. (Logical truth) (Logical truth)

(10) $E$ confirms $H$.

$(9,8$, CCC)

Notice that, in this proof, the crucial step (9) does not appear problematic because, in accordance with (6), $E \vee H$ is not a tautology. Notice also that the conclusion that any observation statement $E$ confirms any hypothesis $H$ such that their disjunction $E \vee H$ is not a tautology is surely unacceptable. Thus, the new proof appears not to be as questionable as Le Morvan's, though just as devastating. In conclusion, if the choice among general confirmation principles is just about (CCC), (SCC) and (EC), it seems in general correct that (CCC) must be rejected. ${ }^{3}$

\section{King's College London, Strand, London WC2R 2LS,UK luca.moretti@kcl.ac.uk}

\section{References}

Glymour, C. 1980. Theory and Evidence. Princeton: Princeton University Press. Hempel, C. 1965. Aspects of Scientific Explanation. New York: Free Press.

Le Morvan, P. 1999. The Converse Consequence Condition and Hempelian qualitative confirmation. Philosophy of Science 66: 448-54.

3 I wish to thank Phillip Meadows for comments and suggestions on early versions of this paper. 